\title{
Endobronchial hemorrhage after intubation with double- lumen endotracheal tube in a patient with idiopathic thrombocytopenic purpura for minimally invasive cardiac surgery
}

-a case report-

\author{
Hee Young Kim, Seung Hoon Baek, Kyoung Hoon Kim, and Nam Won Kim \\ Department of Anesthesia and Pain Medicine, School of Medicine, Pusan National University, Busan, Korea
}

\begin{abstract}
Minimally invasive cardiac surgery (MICS) requires lung isolation. Lung isolation is usually achieved with double-lumen endotracheal tube (DLT). Patients with idiopathic thrombocytopenic purpura (ITP) have an increased risk of bleeding events. We suspected endobronchial hemorrhage after exchange of DLT during induction of anesthesia for replacement of mitral valve in a 62-year-old man with a known ITP. The MICS was stopped and bronchial artery embolization was performed in the angiographic room. In the present case, in order to reduce the risk of bronchial arterial injury in ITP patient we intubated with single lumen endotracheal tube. Lung isolation led to achievement of intermittent total lung deflation. Based on the results, we recommend a high-dose intravenous immunoglobulin therapy and platelet transfusion prior to cardiac surgery in patients with ITP to increase platelet count. Moreover, it is proposed that in order to clear the vision during the operation, ventilation can be held or made intermittent both prior to cardiopulmonary bypass or at its conclusion to permit exposure. (Korean J Anesthesiol 2014; 66: 59-63)
\end{abstract}

Key Words: Cardiac surgery, Double-lumen endotracheal tube, Endobronchial hemorrhage, Idiopathic thrombocytopenic purpura, Minimally invasive surgical procedures.

\footnotetext{
Received: November 21, 2012. Revised: 1st, December 11, 2012; 2nd, January 2, 2013; 3rd, January 9, 2013; 4th, January 14, 2013. Accepted: January 14, 2013.

Corresponding author: Seung Hoon Baek, M.D., Department of Anesthesia and Pain Medicine, School of Medicine, Pusan National University, Beommeo-ri, Mulgeum-eup, Yangsan 626-770, Korea. Tel: 82-55-360-2129, Fax: 82-55-360-2149, E-mail: md.baeksh@gmail.com

(c) This is an open-access article distributed under the terms of the Creative Commons Attribution Non-Commercial License (http:// creativecommons.org/licenses/by-nc/3.0/), which permits unrestricted non-commercial use, distribution, and reproduction in any medium, provided the original work is properly cited.
} 
A double-lumen endotracheal tube (DLT) is usually used as a technique to maintain single-lung ventilation during minimally invasive cardiac surgery (MICS). However, it can lead to bleeding from airway due to the relatively large external diameter and stylet [1]. Airway trauma and bleeding after intubation is a rare complication, but is of importance especially in idiopathic thrombocytopenic purpura (ITP) patients. It is important to manage the airway before and after the treatment.

ITP is an autoimmune disease, which is defined as acquired thrombocytopenia of unknown cause. Strict platelet control should be started before surgery in the case of ITP patients because thrombocytopenia is induced by cardiac surgery due to the influence of extracorporeal circulation [2]. With progression in surgical methods, several cardiac surgeries for patients complicated with ITP have been reported [3,4]. It is recommended to perform a high-dose intravenous immunoglobulin (IVIG) therapy, splenectomy, and platelet transfusion prior to cardiac surgery in ITP patients to increase platelet count $[5,6]$. Consensus has not been reached concerning the selection of therapeutic method and there is no such specific guideline about platelet count concerning the trauma of airway in ITP patients.

In the past, there has been a vast increase in the number of operations using MICS. For operations with MICS, one lung ventilation (OLV) through lung separation is almost essential. The most widely used instrument for lung separation is a DLT [7].

Until date, there has been no case report demonstrating bleeding from airway in ITP patients. In the present report, we present a case of 62-year-old man with known ITP, who had severe mitral regurgitation.

\section{Case Report}

A 62-year-old man (169 cm in height and $69 \mathrm{~kg}$ in weight) was admitted to hospital with dizziness and paresthesia of both hands. He was diagnosed with cerebral infarction, and while doing additional examination, cerebral infarction was assumed to be due to infective endocarditis. Transthoracic echocardiography showed large and hypermobile vegetations on anterior mitral leaflet. He was scheduled to have mitral valve replacement using MICS due to tremendous risk of systemic embolism.

Two months back, he was admitted to the Department of Neurosurgery in our hospital because of subarachnoid hemorrhage in left central sulcus and precentral sulcus, and blood spots on the lower limbs and thrombocytopenia $\left(33,000 / \mathrm{mm}^{3}\right)$ were incidentally detected. Although in a bone marrow aspiration, pathologic finding was not observed and the platelet associated immunoglobulins was negative, ITP was clinically diagnosed. However, steroid therapy was not started immediately and his platelet count was examined as needed.
His platelet count was $40,000 / \mathrm{mm}^{3}$ the day before surgery. Because MICS needed the use of heparin prior to cardiopulmonary bypass (CPB), we decided to transfuse platelets after surgery. For premedication, $0.2 \mathrm{mg}$ of glycopyrrolate was injected intramuscularly 30 minutes before the surgery. Vital signs at the time of arrival at the operation room were blood pressure at $125 / 75 \mathrm{mmHg}$, heart rate at 98 beats/min, and oxygen saturation $\left(\mathrm{SpO}_{2}\right)$ as $99 \%$.

For the induction of anesthesia, while $0.1 \mu \mathrm{g} / \mathrm{kg} / \mathrm{min}$ of remifentanil was infused for three minutes, $10 \mathrm{mg}$ of etomidate and $10 \mathrm{mg}$ of cisatracurium were also injected. Then a leftsided 35 -french DLT was intubated with a stylet. The stylet was removed immediately after the tip of DLT passed through the vocal cords. When the tube was inserted at a depth of $29 \mathrm{~cm}$ to lips, resistance was felt. Tracheal cuff and the bronchial cuff were inflated with $6 \mathrm{ml}$ and $2 \mathrm{ml}$ of air, respectively. After making a judgment that the location of tube was appropriate by using bronchoscope, the bronchial cuff was deflated in order to prevent bronchial injury from postural changes, and there was no bleeding. Anesthesia was maintained with sevoflurane $1.5 \mathrm{vol} \%$, oxygen $1.0 \mathrm{~L} / \mathrm{min}$, medical air $1.5 \mathrm{~L} / \mathrm{min}$ and remifentanil 0.12 $\mu \mathrm{g} / \mathrm{kg} / \mathrm{min}$ were continuously infused intravenously. The patient underwent DLT change when we detected leakage of endotracheal balloon. After the exchange of DLT using tube exchange catheter, we confirmed the location of the DLT by using bronchoscope, and there was no bleeding. Insertion of superior vena cava (SVC) drainage catheter and central venous pressure catheter were performed via right internal jugular vein. Heparin 2,100 IU was administered to prevent clot formation of SVC drainage catheter. There was no sign of bleeding during those procedures. At the end of the procedure, we detected bleeding from the left side of DLT. The appearance of the bleeding was blood-tinged or frothy pink like pulmonary edema. The bleeding was sucked via suction catheter and the amount of the bleeding was about 150$200 \mathrm{ml}$. Due to the bleeding via the left side of DLT, we carried out lung separation immediately and decided to check the location of the bleeding with a fiberoptic bronchoscope. The carinal region and the left and the right bronchus were examined with a fiberoptic bronchoscope, but the focus of the bleeding in the traveling direction from the left mainstem bronchus was not detected. Because fiberoptic bronchoscopic view had poor visibility due to blood, we could not detect the focus of the bleeding and active bleeding from bronchus. Also, there was no bleeding via right side of DLT. Because we could not find the evidence of tracheobronchial laceration or rupture and the patient did not have any subcutaneous emphysema, and pneumomediastium, we excluded the possibility of mechanical injury of airway.

Blood loss was estimated to be $150-200 \mathrm{ml}$ over $30 \mathrm{~min}$. Therefore, we suspected bronchial arterial injury; MICS was immediately stopped and bronchial artery embolization was sched- 
uled in the angiographic room. Vital signs at that time, were blood pressure (BP) at 105/50 $\mathrm{mmHg}$, heart rate (HR) at 115 beats/min, and $\mathrm{SpO}_{2} 89 \%$. During the transportation of the patient to the angiographic room, gradual decrease in the bleeding was noted, and the patient's vital signs in the angiographic room were stable (BP at 110-120/50-80 mmHg, HR 100 beats/min, and $\mathrm{SpO}_{2}$ 99-100\%). Angiography of the bronchial artery was performed, but focus of the bleeding was not found (Fig. 1). With reference to the risk of re-bleeding from the injured bronchial artery and the possibility of uncontrolled bleeding in patient with ITP, we decided to embolize the contributing bronchial arteries. After finishing the embolization of bilateral bronchial arteries, the chest X-ray was checked, and total atelectasis of left lung was observed (Fig. 2). There was no evidence of active bleeding, but

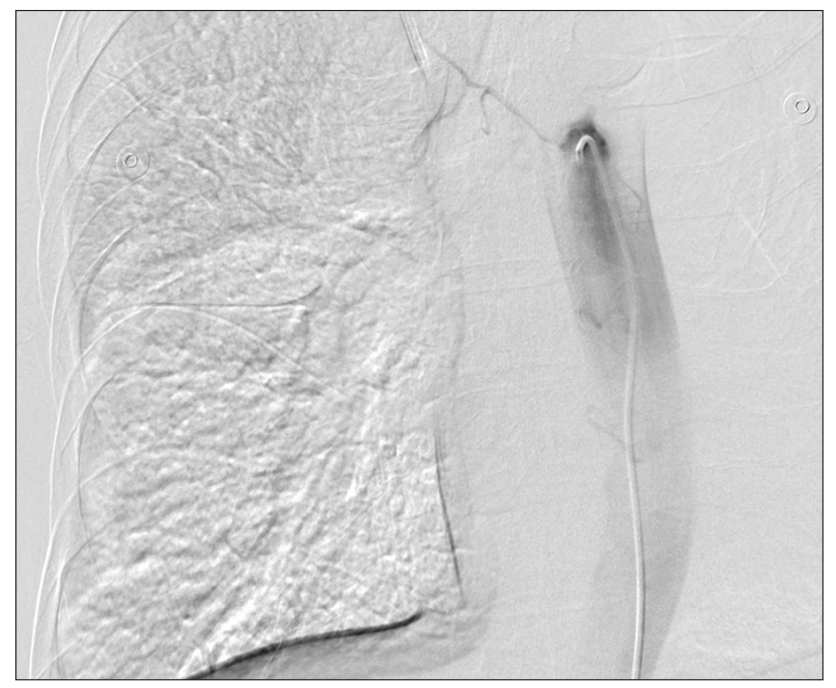

Fig. 1. Bronchial arterial angiography shows the absence of bleeding event. blood clot was detected and removed using bronchoscope. After the removal of blood clot, an improvement in lung collapse was noted.

The patient was scheduled for MICS 6 days later. Due to lowered platelet count $\left(54,000 / \mathrm{mm}^{3}\right)$, a high-dose IVIG therapy ( $35 \mathrm{~g} /$ day, $0.5 \mathrm{~g} / \mathrm{kg}$ ) for 2 days was started. Platelet count was increased to $87,000 / \mathrm{mm}^{3}$ after 6 days. We intubated with single lumen endotracheal tube and lung isolation was achieved with intermittent total lung deflation. The surgery was done safely, and the outcome was favorable for the patient without any postsurgical decrease in platelet count or bleeding. He was extubated on the postoperative day (POD) 2, and transferred from the intensive care unit to the ward on POD 5. The patient left the hospital after making a full recovery on POD 28.

\section{Discussion}

ITP is an autoimmune coagulation disorder characterized by a decreased number of circulating platelets and a shortened platelet life span. Generally, platelet count is very low in conditions of ITP, often $50,000 / \mathrm{mm}^{3}$ or less [8]. Although successful cardiac operations have been reported in ITP patients, large quantities of platelets, or blood transfusions were necessary in the postoperative period [9]. High-dose IVIG therapy is recognized to be very effective in ITP patients. Platelet counts usually increase in the consecutive 5 to 7 days after administration of high-dose IVIG and subsequently are restored to the previous level within a month's time [10]. Splenectomy is usually considered as a second line treatment for ITP patients. A published work reports on 23 patients with ITP, who safely underwent a cardiac surgical procedure, moreover no platelets were transfused in 3 patients. All 3 patients received preoperative treatment with IVIG but no prophylactic splenectomy [4]. Koyanagi
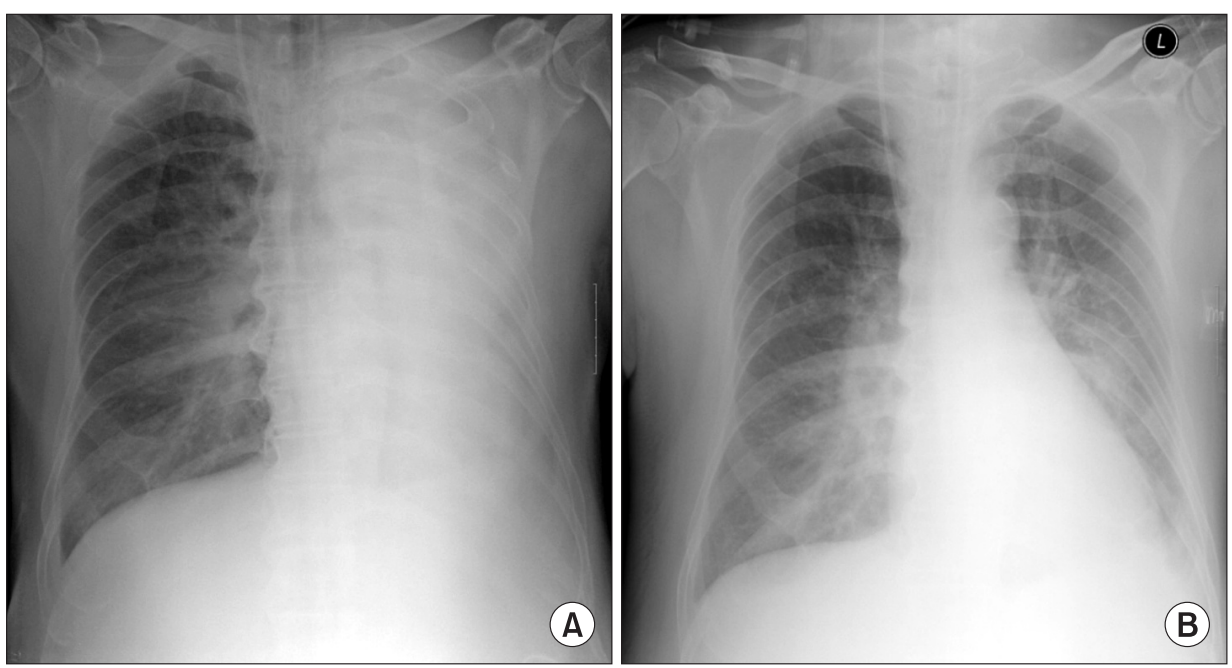

Fig. 2. Chest X-ray. (A) The chest X-ray shows total atelectasis of left lung after concluding the embolization of bilateral bronchial artery. There was no evidence of active bleeding, but blood clot was detected and removed using bronchoscope. (B) After the removal of blood clot, an improvement in lung collapse was observed. 
et al. achieved mitral valve replacement in ITP patient at redo operation using neither allogenic platelet nor blood transfusion throughout the whole perioperative course, by means of preoperative high-dose IVIG and simultaneous splenectomy [4].

Although platelet count was low by $63,000 / \mathrm{mm}^{3}$ in the preoperative laboratory study in our patient, considering economic status of the patient and based on the Koyanagi et al. case report, transfusion of platelets and IVIG therapy was not started before surgery. However, it is recommended to perform a highdose IVIG therapy, splenectomy, and platelet transfusion prior to cardiac surgery in ITP patients to increase platelet count [5, 6]. Consensus has not been reached concerning the selection of therapeutic method, but surgery may be performed when platelet count is increased following various therapies in case of elective surgery for a patient with stable pre-surgery hemodynamics.

In the past several years, there has been an increase in operations using MICS. Because most of the MICS uses a right thoracotomy, lung isolation has been a mainstay of anesthetic management. The method of choice for achieving lung isolation is also debatable. The most common techniques for achieving lung isolation are left-sided DLT or right-sided bronchial blocker placement. Once positioned, there are definite benefits of lung isolation as they are less likely to be dislodged than bronchial blockers and they allow for the application of continuous positive airway pressure and suction to the deflated lung. Therefore, most of the anesthesiologists use DLT for lung isolation in MICS. However, DLT may be more challenging to place in certain patients and may be associated with airway trauma and bleeding [11]. There is no consensus associated with control of platelet count for reducing the airway trauma and bleeding in ITP patients. Based on our experience, increasing the platelet count by high-dose IVIG therapy or platelet transfusion is helpful to reduce the complication associated with airway in ITP patient. Also, OLV is not mandatory during the conduct of MICS. Ventilation can be held or made intermittent both prior to $\mathrm{CPB}$ or at its conclusion to permit exposure. $\mathrm{CPB}$ can also be initiated prior to exposing the left atrium at the beginning of the op- eration, although this approach may unnecessarily increase the total CPB time. If the anesthesiologists consider the risk of airway trauma or bleeding in ITP patients, they could choose the ventilation method; single lumen endotracheal tube with Arndt bronchial blocker, univent tube under fiberoptic bronchoscope guidance or intermittent single-lung ventilation as per the need of the surgeon [11].

Our patient was taken up for emergency embolization of the bilateral bronchial arteries. It is rare to identify frank arterial extravasation as the definite source of hemorrhage during angiography for hemoptysis. It is difficult to be sure that the vessel that is embolized is actually the source of hemorrhage and theoretically every possible vessel contributing to bronchial artery supply should be embolized. Indications for bronchial artery embolization include massive hemoptysis, recurrent hemoptysis, and to control bleeding temporarily before surgery [12].

In conclusion, we recommend performing a high-dose IVIG therapy and platelet transfusion prior to cardiac surgery in patients with ITP to increase platelet count. If the platelet count is increased, it may reduce the complications of airway management. Also, in order to reduce the risk of bronchial arterial injury in ITP patients, lung isolation can be achieved by single lumen endotracheal tube with Arndt bronchial blocker or univent tube under fiberoptic bronchoscope guidance. If OLV is not mandatory for performing MICS, ventilation can be held or made intermittent both prior to $\mathrm{CPB}$ or at its conclusion to permit exposure. Anesthesiologists must carefully observe the progress of patients after the operation, and if there are suspicious symptoms or signs, adequate diagnosis and quick treatment should be done considering the possibility of bleeding from airway.

\section{Acknowledgments}

This work was supported by a 2-Year Research Grant of Pusan National University.

\section{References}

1. Liu H, Jahr JS, Sullivan E, Waters PF. Tracheobronchial rupture after double-lumen endotracheal intubation. J Cardiothorac Vasc Anesth 2004; $18: 228-33$.

2. Campbell FW, Edmunds LH. Platelet function and cardiopulmonary bypass. In: Cardiopulmonary bypass. Edited by Gravlee GP, Davis RE, Utley JR: Meryland, Williams \&Wilkins. 1993, pp 407-35.

3. Sezai A, Akiyama K, Fukushima S, Kashiwazaki S, Shiono M, Horikoshi A, et al. A surgical case of mitral regurgitation due to active infective endocarditis with idiopathic thrombocytopenic purpura. Ann Thorac Cardiovasc Surg 2011; 17: 618-23.

4. Steven MN, James BE, Edmond C. Anesthesia for thoracic surgery. In: Clinical anesthesia. 6th ed. Edited by Barash PG, Cullen BF, Stoelting RK, Cahalan MK, Stock MC: Wolters Kluwer, Lippincott Williams\& Wilkins. 2009, pp 1042-3.

5. Inoue Y, Lim RC, Nand P. Coronary artery bypass grafting in an immune thrombocytopenic purpura patient using off-pump techniques. Ann Thorac Surg 2004; 77: 1819-21. 
6. Koyanagi T, Kyo S, Hirooka E, Koyama I, Omoto R. Redo without transfusion in a patient with idiopathic thrombocytopenic purpura. Ann Thorac Surg 2000; 69: 1261-3.

7. Ohno H, Higashidate M, Yokosuka T. Washing of the residual solution of cardiopulmonary bypass circuit after coronary artery bypass grafting in idiopathic thrombocytopenic purpura. J Cardiovasc Surg (Torino) 2002; 43: 185-8.

8. Marõnas JM, Llamas P, Caffarena JM. Mitral valve replacement and splenectomy in a patient with chronic idiopathic thrombocytopenic purpura. Thorac Cardiovasc Surg 1982; 30: 407-8.

9. Mathew TC, Vasudevan R, Leb L, Pezzella SM, Pezzella AT. Coronary artery bypass grafting in immune thrombocytopenic purpura. Ann Thorac Surg 1997; 64: 1059-62.

10. Mori Y, Hadama T, Takasaki H, Oka K, Shigemitsu O, Miyamoto S, et al. Aortic valve replacement and splenectomy in a patient with chronic idiopathic thrombocytopenic purpura-preoperative management with high-dose g-globulin. Heart Vessels 1991; 6: 121-4.

11. Vernick WJ, Woo JY. Anesthetic considerations during minimally invasive mitral valve surgery. Semin Cardiothorac Vasc Anesth 2012; 16: 11-24.

12. Andersen PE. Imaging and interventional radiological treatment of hemoptysis. Acta Radiol 2006; 47: 780-92. 